\title{
Gas chromatography in the chemical and pharmaceutical industries
}

\author{
W. Luijten \\ Department of Structural Analysis, Technologie Servier, BP. 1749, 45007 Orléans Cedex 1, France
}

\begin{abstract}
In the development and manufacture of chemical and pharmaceutical products analytical chemistry plays a vital role in the quality control of the intermediate and final products. Separation methods occupy an important place in the array of available analytical techniques. Depending on the nature of the compounds, gas chromatography methods continue to be used to a large extent, especially in automated routine controls. The use of specialised injection and detection methods has further increased its field of applications.
\end{abstract}

survey of the literature concerning the various separation methods shows that a large majority of the contributions focus on separations that take place in the liquid phase. Over the past ten years, new and existing techniques and combination of techniques involving liquid chromatography in its various forms have become available to the chemical and pharmaceutical industries.

The steady growth of these techniques is partly due to the increasing complexity of the chemical entities being developed, urging separation and analytical scientists to think of new ways to separate and characterise chemical compounds. In addition, the growing demand for the pharmaceutical industry to produce more data, both in quality and in quantity, has had a major impetus in developing automated and combined analyses of active drug substances in various matrices. This focus on liquid phase separation methods tends to obscure the fact that other separation methods, such as gas chromatography, are still widely in use. One reason that can be heard occasionally during symposia is that one does not talk much about techniques that do the job...

Gas chromatography is a relatively old technique, which is well documented in the literature.

This contribution will therefore concentrate on a number of applications involving gas chromatography in combination with specific injection and detector systems. The techniques discussed hereafter cover a wide spectrum of chemical analyses, from the detection of known or unknown impurities in compounds or in drug substances to analytical assays for pharmacokinetic studies.

\section{Residual solvent analysis}

The manufacture of a drug substance, or any chemical entity, involves large quantities of solvents. Solvents may be used at any step of the synthetic pathway, but their use occurs frequently in the last purification step. The directives concerning medicinal products demand the development of initial and routine analytical controls for any active substance in a drug.

Amongst these tests is a check for residual solvents. Gas chromatography has proven to be a very efficient method of choice for the analysis organic solvents. This technique has been successfully employed with a variety of detector systems, depending on the solvents to be analysed. Samples may be introduced by direct injection (split/splitless) of the dissolved drug substance. However, this method has the inconvenience of a rapid decrease in column efficiency due to on-column retention of non-volatile components. Sampling devices such as automated head-space injectors present the advantage of limiting the injection to volatile compounds. Furthermore, due to the enrichment of the volatile components in the gas phase, head-space sampling gives generally better sensitivities than the direct injection of a solution [1].

The use of small dedicated mass spectrometers as sophisticated detector systems increases the specificity and therefore the correct assignment of solvents to their corresponding chromatographic peaks. A typical set-up used for the routine determination of organic solvent content in drug substances consists of an automated head-space injector (Tekmar 7000/7050), a gas chromatograph (Hewlett Packard 5890) and a bench-top mass spectrometer (Finnigan ITD 800). This fully integrated system uses a fused silica capillary column of $25 \mathrm{~m}$ length and $0.32 \mathrm{~mm}$ internal diameter. The solid phase is a polyethylene glycol polymer, with a film thickness of $1.23 \mathrm{~mm}$ (Chrompack CPWAX 52CB). Compounds may be analysed either in solid form or by dissolution in a suitable non-volatile solvent (i.e. water, DMSO, glycol, diglyme). After desorption from the sample matrix by static or dynamic head-space concentration (depending on the desired detection level) [2], the evaporated solvents are injected onto the capillary column.

When the presence of organic solvents is not known in advance, the mass spectrometer allows the recognition of organic solvents through the use of appropriate search and identification software. Once the solvent profile of a compound is known, routine quantitative analysis using reference compounds and quantification software can be carried out, following a fully automated sequence of events.

To illustrate this, the following table presents the results obtained from a number of solvents dissolved in diglyme, in comparison with the maximum authorised levels in drug substances [3]. The test quantities are equivalent to the dissolution of $60 \mathrm{mg} \mathrm{ml}^{-1}$ of drug substance in the case of methanol, acetonitrile, toluene and isopropanol. For 
Table I. Residual solvent content.

\begin{tabular}{|c|c|c|c|}
\hline Solvent & $\begin{array}{c}m g m t^{-1} \\
\text { a) }\end{array}$ & $\begin{array}{c}\text { ppm } \\
(\text { Ph. Eur.) }\end{array}$ & $\begin{array}{l}\text { ppm } \\
\text { b) }\end{array}$ \\
\hline methanol & 60 & 1000 & 4350 \\
\hline dichloromethane & 90 & 500 & 1675 \\
\hline acetonitrile & 3 & 50 & 150 \\
\hline chloroform & 9 & 50 & 55 \\
\hline toluene & 60 & 1000 & 3000 \\
\hline isopropanol & 30 & 500 & 1750 \\
\hline dioxan & 10 & 100 & 180 \\
\hline
\end{tabular}

a) concentration corresponding to the maximum allowed level,

b) results for the analysis of $5 \mathrm{ml}$ of solution, extraction at $100{ }^{\circ} \mathrm{C}$ for $20 \mathrm{~min}$.

dichloromethane, chloroform and dioxan the respective quantities are 150, 180 and $100 \mathrm{mg} \mathrm{ml}^{-1}$.

Tests performed in other matrices (DMSO, ethyleneglycol) showed similar results, with individual differences due to the polarity of both solvent and matrix. Better evaporation of the solvent from the matrix is generally achieved when the polarities of solvent and matrix differ by a greater extent.

Detection levels of down to $25 \mathrm{ppm}$ are readily attainable through the careful choice of parameters such as: the amount of compound to dissolve, a solvent matrix adapted to the solvents under analysis and the head-space concentration conditions.

\section{Freon and freon substitute impurities}

Since the ban on the use of certain freons, alternative propellant gases have been developed over the past few years.
Besides the domestic use of these propellants in various sprayed articles (deodorants, hair sprays, paints) the pharmaceutical industry is interested in these gases as new propellants for the delivery of drug substances from metereddose inhalers, which are frequently used to treat diseases of the respiratory tract (asthma, bronchitis).

One of these newly developed propellant gases is HFA 134a (1,1,1,2-tetrafluoroethane). As for new drug substances, new pharmaceutical excipients have to undergo a series of chemical and toxicological tests before they can be accepted as such. Part of the evaluation process concerns their chemical property and more specifically, their impurity profile. Since potential impurities in HFA 134 a may be expected to be in the same volatility range as the main compound, gas chromatography in combination with mass spectrometry has been used to assess its chemical purity. The column used in these analyses must be able to separate several highly volatile compounds to obtain reliable mass spectra. In this particular case a styrene/vinylbenzene polymer PLOT column (PoraPLOT Q, Chrompack) of $12.5 \mathrm{~cm}$ length and 0.25 $\mathrm{mm}$ ID was used.

The film thickness was $10.0 \mathrm{~mm}$ to increase the sample capacity needed to detect small amounts of impurities. Injection was performed using a $0.5 \mathrm{ml}$ gas-tight syringe and a split injector (gas split $60 \mathrm{ml} \mathrm{min} \mathrm{m}^{-1}$ ). The sample was taken directly from the liquid phase in the HFA 134a container. Helium was used as carrier gas at a linear gas velocity of $55 \mathrm{~cm} \mathrm{sec}^{-1}$, whilst the temperature of the column was kept at $50{ }^{\circ} \mathrm{C}$ for $2 \mathrm{~min}$, then raised to $200{ }^{\circ} \mathrm{C}$ at $25^{\circ} \mathrm{C} \mathrm{min}{ }^{-1}$. The column was lead through the $\mathrm{GC} / \mathrm{MS}$-interface $\left(200{ }^{\circ} \mathrm{C}\right)$ directly into the source of the mass spectrometer ( $\mathrm{VG}$ Analytical 70-VS). Electron impact mass spectra were obtained at $70 \mathrm{eV}$.

Since the levels of the impurities in HFA 134a were expected to be in the $1-100 \mathrm{ppm}$ range, relatively large quantities of HFA 134a had to be injected. This results in considerable peak tailing of the principal compound, which can obscure low level peaks eluting just after the main compound, when non-specific detectors are used. However, the

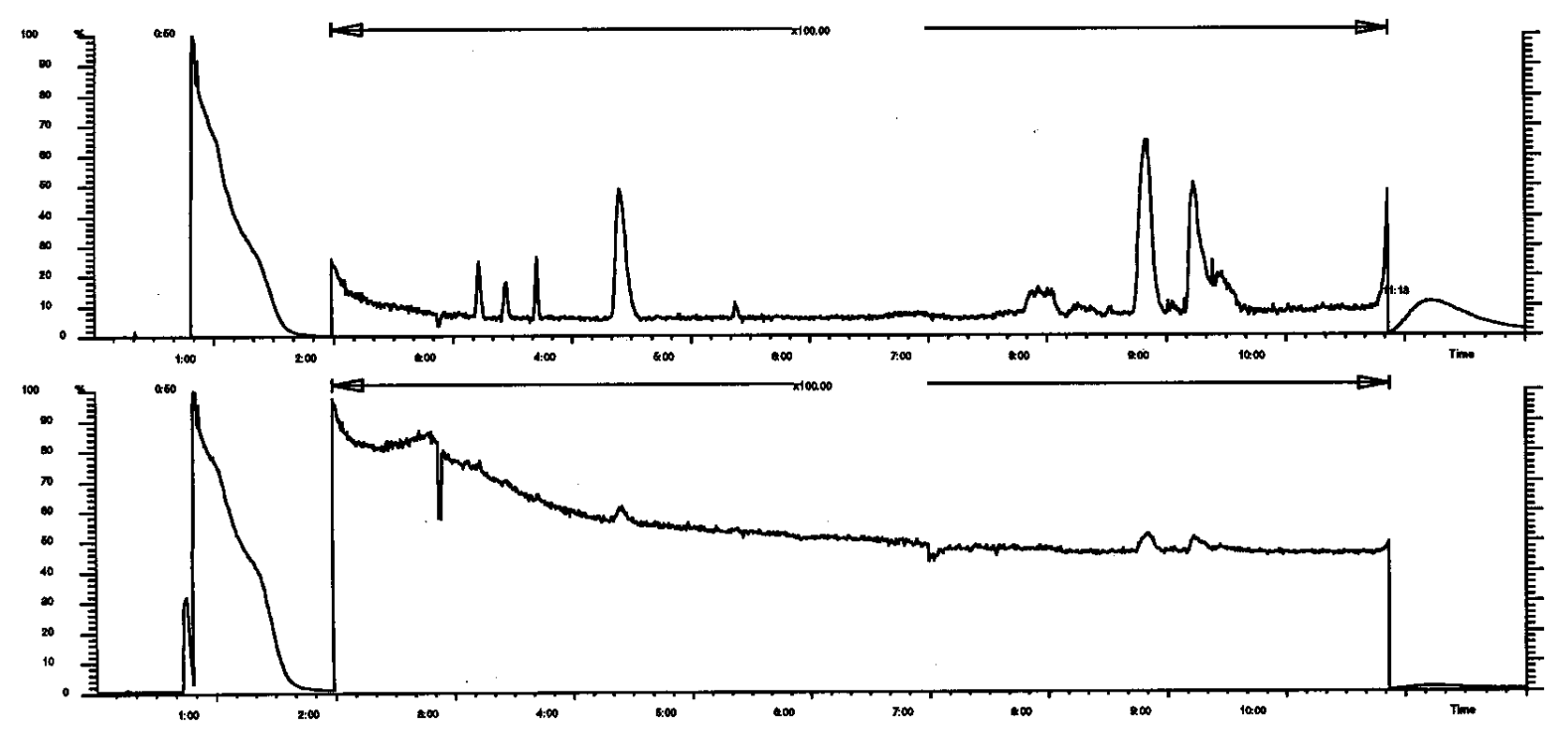

Figure 1. Ion chromatograms, (top) before subtraction of main compound fragment ions and (bottom) after subtraction. 
mass spectrometer allows the subtraction of the major fragment ions of HFA 134a from the mass spectra in the chromatogram (Fig. 1), making it possible to obtain interpretable mass spectra.

Unambiguous identification of the impurities is straightforward, using reference compounds and the available electron impact mass spectral libraries [4].

Table II. HFA 134a impurities.

\begin{tabular}{cc}
\hline Retention time & Compound \\
\hline $3: 13$ & 1-chloro-2,2-difluoroethene \\
$3: 27$ & 1-chloro-1,2-difluoroethene \\
$3: 42$ & 1-chloro-2-fluoroethene \\
$4: 24$ & 1,1 -dichloro-2,2,2-trifluoroethane \\
$5: 25$ & trichlorofluoro methane \\
$7.50-8.50$ & xylenes \\
$8: 50$ & phenol \\
$9: 28$ & benzaldehyde \\
\hline
\end{tabular}

\section{GC with infrared detection}

An interesting combination of gas chromatography with another spectral technique is GC/FT-IR. Infrared spectroscopy is widely used as a routine control in chemical synthesis. Both at the chemical production plant, as well as during the manufacture of the final drug product, both drug substances and excipients are routinely checked by infrared spectroscopy. Although infrared spectroscopy is an integral part of the official document demonstrating the chemical structure of a (new) drug substance, the technique is much less frequently used in structure elucidation of unknown compounds. The example below illustrates an application where the combination of gas chromatography and FT-IR proved its value in distinguishing a pair of isomers [5].

The synthesis of a specific metabolite of a drug substance under pre-clinical development included the introduction of an aldehyde substituent on 2,3-dichlorophenol, leading to one of the two possible isomers:<smiles>O=Cc1ccc(O)c(Cl)c1Cl</smiles><smiles>O=Cc1ccc(Cl)c(Cl)c1O</smiles>

GC/MS and NMR analyses of the isolated product were unable to determine the unambiguous identification of the resulting compound. GC/FT-IR was thus used to obtain an infrared spectrum of the reaction product in the gas phase
(Fig. 2, top). The presence of two strong absorption bands at $1712 \mathrm{~cm}^{-1}$ (carbonyl) and $1592 \mathrm{~cm}^{-1}$ (def -COH) and a weak absorption band at $3560 \mathrm{~cm}^{-1}$ (stretch -OH) strongly suggested the aldehyde substituent para to the hydroxyl group. In the absence of strong intermolecular hydrogen bonding in the gas phase, one would expect the absorption bands at $3560 \mathrm{~cm}^{-1}$ and $1592 \mathrm{~cm}^{-1}$ to be practically invisible in the ortho isomer owing to intramolecular hydrogen bonding between the hydroxyl and carbonyl substituents. This difference in behaviour is only observable in the gas phase because of strong intermolecular hydrogen bonding in the liquid phase. This hypothesis was verified by the GC/FT-IR analysis of the commercially available reference compounds para- and ortho-hydroxy benzaldehyde. The infrared spectrum of the para-isomer showed the expected absorption bands at $3600 \mathrm{~cm}^{-1}$ and $1605 \mathrm{~cm}^{-1}$ besides the carbonyl band at $1718 \mathrm{~cm}^{-1}$ (Fig. 2, middle), whilst the ortho-isomer only showed a strong carbonyl band at $1692 \mathrm{~cm}^{-1}$ (Fig. 2, bottom), shifted to lower wavenumber due to hydrogen bonding. Comparison with the infrared spectrum of the synthetic product confirmed the latter to be the para-isomer.

\section{Pharmacokinetic assay}

Two simultaneous developments in mass spectrometry have led to an explosive growth of the use of mass spectrometry in the biological and biochemical sciences: the invention of the electrospray ionisation source and the appearance of various MS/MS mass spectrometers. Electrospray ionisation makes the coupling of liquid chromatography with mass spectrometry relatively straightforward, while tandem mass spectrometry allows highly specific detection of target compounds in complex matrices. Particularly in the pharmaceutical industry, pharmacokinetic development is now being conducted with an ever increasing number of LC/MS and LC/MS/MS methods.

Occasionally however, gas chromatography coupled to mass spectrometry can still be found useful for pharmacokinetic assays, when the drug substance and major metabolites are both sufficiently volatile. The relative ease of operation of modern GC and GC/MS instruments makes them attractive tools for the assays of large numbers of samples using automated procedures [6].

Piribedil is the active substance of Trivastal ${ }^{\circledR}$, a dopaminergic receptor agonist, used in the treatment of central nerve system disorders. Recently, a new drug formulation was developed, in which a pharmacokinetic evaluation study was carried out, using GC/MS.

Sample preparation consisted of liquid-liquid extraction of $1 \mathrm{ml}$ of plasma, to which was added an internal standard (deuterated piribedil), centrifugation and evaporation to dryness. The sample was taken up in $25 \mathrm{ml}$ of acetonitrile and $2 \mathrm{ml}$ of the resulting solution were injected onto the column. The gas chromatograph (Hewlett Packard 5890) was equipped with an automated split-splitless injector (operating in the splitless mode) and a $10 \mathrm{~m}$ fused silica capillary column of $0.25 \mathrm{~mm}$ internal diameter. The stationary phase was $100 \%$ methyl siloxane with a film thickness of $0.12 \mathrm{~mm}$ (Chrompack CPSil 5CB). Helium was used as carrier gas at a head pressure of $60 \mathrm{kPa}$. The injector and the 

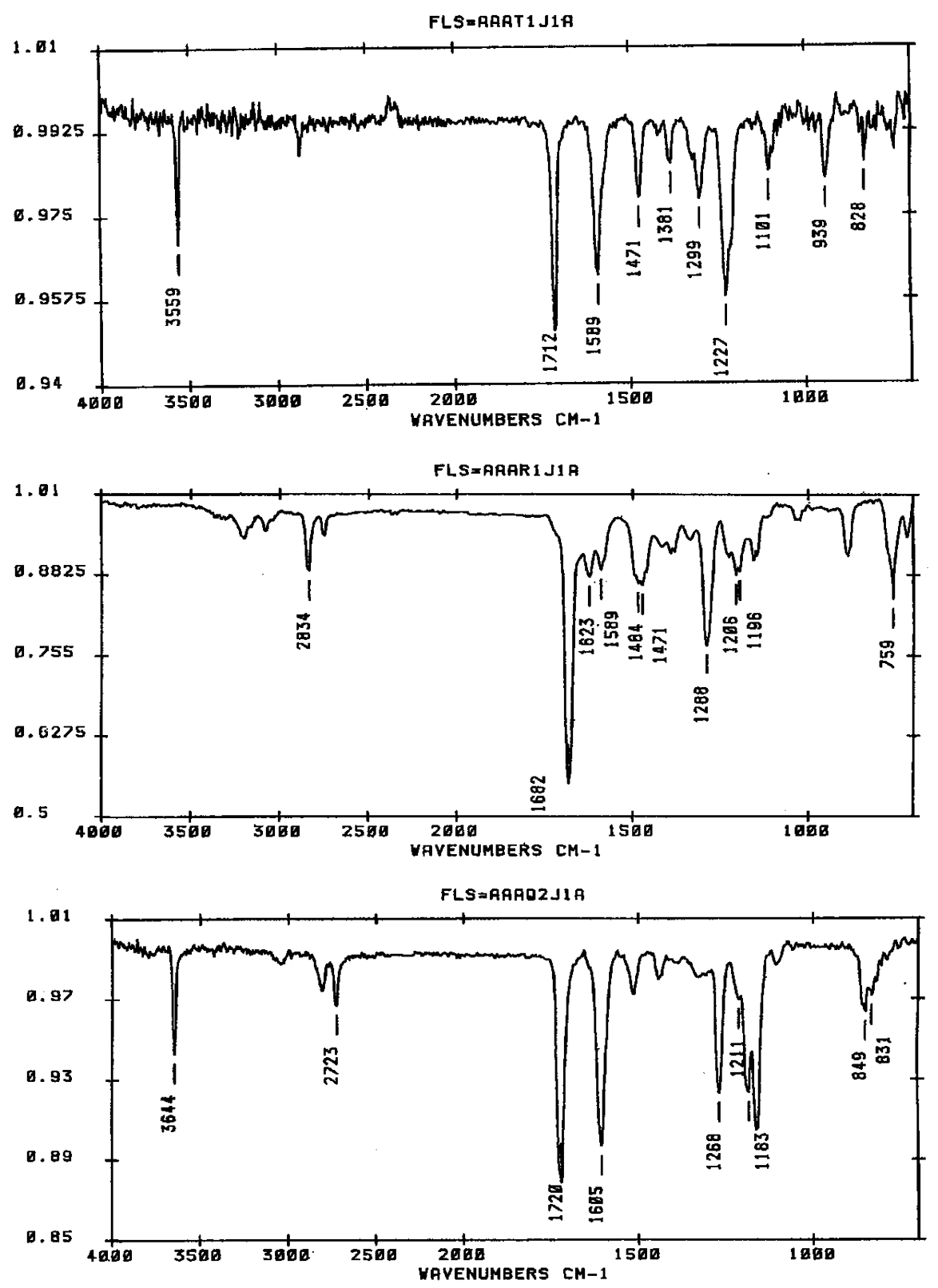

Figure 2. Infrared spectra of (top) unknown isomer; (middle) ortho-hydroxybenzaldehyde and (bottom) para-hydroxybenzaldehyde.

MS-interface were kept at $270{ }^{\circ} \mathrm{C}$ and $300{ }^{\circ} \mathrm{C}$, respectively. The temperature gradient of the $\mathrm{GC}$-oven started at $120^{\circ} \mathrm{C}$ and was raised to $320{ }^{\circ} \mathrm{C}$ at $25^{\circ} \mathrm{C} \mathrm{min}^{-1}$.

\section{Piribedil}<smiles>[CH]1Oc2ccc(CN3CCN(c4ncccn4)CC3)cc2O1</smiles>

\section{* deuterium labelled for internal standard}

Under these conditions the retention times for both compounds were 4.55 min. Mass spectrometry (Hewlett Packard
5970A) was performed in the EI mode (35 eV), using selective ion monitoring of the molecular ions of piribedil and the internal standard $(\mathrm{m} / \mathrm{z}, 298$ and $\mathrm{m} / \mathrm{z}, 300)$. No interfering signals at these masses could be detected in the blank samples.

The method has been validated for an overall concentration range of 0.1 to $100 \mathrm{ng} \mathrm{ml}^{-1}$, at two distinct concentration ranges, 0.10 to $5.0 \mathrm{ng} \mathrm{ml}^{-1}$ and 0.5 to $25 \mathrm{ng} \mathrm{ml}^{-1}$, using 1 and $2.5 \mathrm{ng} \mathrm{ml}^{-1}$ of internal standard, respectively. Although an HPLC (UV) method was also developed, the final choice to use GC/MS was determined by the speed of the analysis (only 15 min per injection), the specificity of the detection and the relative ease of the sample preparation, which could be highly automated.

The choice whether to use specific detectors, such as electron capture detectors (ECD) or mass spectrometry, may 
largely depend on the nature of the analytes and on the desired detection levels. When using ECD or mass spectrometry in the negative chemical ionisation mode, detection levels may drop significantly with respect to the more classical methods that rely on FID or electron impact [7]. If the compound is not particularly sensitive to electron capture, one may solve this problem by including a suitable derivatisation step, introducing fluorinated acyl groups onto hydroxyl or amine groups. As in LC/MS, when using GC/MS, suitably deuterated analogues are frequently used as internal standards, since the chromatographic behaviour of these compounds is almost identical to their non-deuterated counterparts.

\section{Conclusion}

Despite the preponderance of liquid chromatography methods in chemical analysis, gas chromato graphy still merits its place in the arsenal of separation methods in the industrial analytical laboratories. Although new developments in gas chromatography are less frequent, the technique has matured to such an extent that its operation has become routine in the laboratory. The judicious choice of specific injection and detection systems has extended the use of gas chromatography to a wide range of applications. The decision whether to use gas chromatography or other separation methods (HPLC) is of course determined by the volatility of the analytes. However, this choice may be influenced by a careful inspection of the overall analytical sequence, from sample preparation to the final calculations. Ease of operation, reliability, robustness of the method, automation, cost of gases, solvents and reagents, sample throughput and speed of analysis are all important criteria to the industrial analytical laboratories.

\section{References}

1. Nunez, A. J.; Gonzales, L. F.; Janak, J. J. Chromatogr. 1984, 300, 127-162.

2. Piet, G. J.; Luijten, W.; Van Noort, P. In: Environmental car cinogens, selected methods of analysis; Fishbein, L.; O'Neill, I. K., Eds., IARC, Lyon, 1985, 7; pp 319-343.

3. Tinto, A. Pharmeuropa 1994, 4(2), 151.

4. McLafferty, F. W.; Stauffer, D. B., The Wiley/NBS Registry of Mass Spectral Data, Wiley \& Sons, New York, 1989.

5. Damien, G. Spectra 2000 1991, 158, 29-35.

6. Fourtillan, J. B.; Gobin, P.; Faye, B.; Girault, J. Biol. Mass Spectrom. 1994, 23, 499-509.

7. Reimer, M. L. J.; Mamer, O. A.; Zavitsanos, A. P; Siddiqui, A. W.; Dadgar, D. Biol. Mass Spectrom. 1993, 22, 235-242. 